\title{
A ATUAÇÃO DO ENFERMEIRO AUDITOR FRENTE ÀS CONTAS HOSPITALARES DO SISTEMA ÚNICO DE SAÚDE
}

\section{ARTIGO ORIGINAL}

SILVA, Josilene Braz da ${ }^{1}$, ZACARIAS, Ezequiel de Souza², SANTOS, Everaldo Almeida dos ${ }^{3}$, SILVA, Milena Nascimento da ${ }^{4}$

SILVA, Josilene Braz da. Et al. A atuação do enfermeiro auditor frente às contas hospitalares do sistema único de saúde. Revista Científica Multidisciplinar Núcleo do Conhecimento. Ano 06, Ed. 09, Vol. 02, pp. 123-133. Setembro de 2021. ISSN: 2448-0959, Link de acesso:

https://www.nucleodoconhecimento.com.br/saude/contas-hospitalares, DOI: 10.32749/nucleodoconhecimento.com.br/saude/contas-hospitalares

\section{RESUMO}

O presente artigo é o resultado de uma pesquisa bibliográfica que tem como questão norteadora: Quais são as principais evidências literárias sobre a Atuação do Enfermeiro auditor frente às contas hospitalares do SUS? Além de identificar as tomadas de decisões dos setores administrativos e gerências que administram o ambiente hospitalar. Diante das atividades hospitalares é preciso avaliar os bens, despesas e custos relacionados a manutenção e funcionamento dos setores hospitalares. A avaliação da auditoria durante o processo das atividades diárias dos colaboradores é fundamental para o controle dos recursos direcionados para a manutenção e atendimento dos pacientes e clientes. O papel do enfermeiro auditor requer que estes estejam qualificados e possuam conhecimentos ao fiscalizar os hospitais do Sistema Único da Saúde. Essa pesquisa trata-se de uma revisão literária na qual foram selecionados materiais publicados em um período de dez

\footnotetext{
${ }^{1}$ Graduação em Administração.

${ }^{2}$ Graduado em letras - Português e Inglês.

${ }^{3}$ Curso de nível superior Bacharel Enfermagem.

${ }^{4}$ Graduação em Psicologia.
}

RC: 96819

Disponível em: https://www.nucleodoconhecimento.com.br/saude/contashospitalares 
anos (2009 a 2018). Dentre os títulos pesquisados, foi possível selecionar um total de 14 artigos. Portanto, conclui-se que, embora a auditoria tenha se tornado parte do processo organizacional empresarial, ao longo dos anos observou-se que a auditoria hospitalar ainda está longe de ser tratada como por parte do cenário da saúde atual. Entretanto, a auditoria hospitalar está se aprimorando para que o futuro possa ser reconhecido como instrumento de melhoria contínua com intenção de prestar assistência aos principais registros realizados nos setores hospitalares.

Palavras-chave: Sistema Único da Saúde, Fiscalização, Enfermeiro.

\section{INTRODUÇÃO}

Historicamente ao reportar a auditoria é preciso explanar alguns fatores, na qual, fornecem aspectos gerências, financeiros e contábeis. Diante disto, o presente artigo tem como questão norteadora: quais são as principais evidências literárias sobre a Atuação do Enfermeiro auditor frente às contas hospitalares do SUS? Para isso, é fundamental comprovar as adequações que aciona determinadas situações no ambiente empresarial, sejam eles na atividade profissional, como na área pública ou privada. Isto é, a auditoria realizada por qualquer pessoa que se utiliza do conhecimento de auditor para analisar minuciosamente as informações contraídas. Nesse sentido, o enfermeiro auditor tem a função de analisar documentos da equipe de enfermagem antes e depois dos procedimentos, além disso, está atento ao cotidiano dos enfermeiros, buscando está em constante interação com os outros enfermeiros. Contribuindo, na análise de prontuários das pessoas enfermas, liberação dos pacientes, contribui na orientação de medicação aos pacientes, sendo estas algumas tarefas do enfermeiro auditor.

O enfermeiro auditor pode ser compreendido como um gerenciador dos serviços da saúde e liderar seus companheiros de trabalho. No entanto, o enfermeiro auditor é responsável também pela auditoria, isto é, por existir um perfeito funcionamento das unidades de saúde, sejam elas públicas ou privadas, ou seja, proporcionando o serviço de qualidade ao paciente ou cliente.

RC: 96819

Disponível em: https://www.nucleodoconhecimento.com.br/saude/contashospitalares 
Por conseguinte, a auditoria é imprescindível para organização e planos no que concerne a atuação da saúde, assim como, cumprimento e gerenciamento das atividades prestadas pelos enfermeiros dentro da Instituição de Saúde. Possivelmente, a auditoria de enfermagem é uma avaliação administrativa indispensável para a fundação ao que é classificado como sistematização da assistência de enfermagem pública ou privada.

Portanto, em conformidade com o autor Motta (2010), o enfermeiro auditor necessita atuar nos padrões estabelecidos pela ética e termos legais, sendo assim, manipular e compreender o elo entre hospitais prestadoras de planos de saúde, pois, para Mota (2010) é preciso entender e se atualizar constantemente, conhecer os assuntos da medicina que sofrem mudanças corriqueiramente, ou seja, conforme avança a tecnologia humana, a medicina também necessita acompanhar seu desenvolvimento, com intuito de servir o homem moderno. Além disso, o enfermeiro auditor é responsável por gerenciar determinadas negociações, possuir argumentos sólidos e baseados em fatos. Dessa forma, mostrando seu desempenho e agilidade, em suma, o enfermeiro auditor é o indivíduo capaz de exercer atividades administrativas com máxima excelência e profissionalismo, qualificando-se e ressaltando sua importância na área da saúde.

\section{O ENFERMEIRO AUDITOR E SUA PARTICIPAÇÃO NA FISCALIZAÇÃO DAS CONTAS HOSPITALARES DO SISTEMA ÚNICO DE SAÚDE}

O procedimento na auditoria hospitalar é executado pelo enfermeiro classificado como especialista, isto é, devido ser conhecido a necessidade da existência dos enfermeiros, o trabalho desse profissional no meio social é relevante e preciso, sendo assim seu trabalho deve ser valorizado, assim como sua qualificação deve ser constantemente assegurada, com 0 intuito de proporcionar excelentes profissionais na área da enfermagem, pois o enfermeiro viabiliza para a sociedade um trabalho eficaz aos pacientes. Sendo assim, no ponto de vista de Kurcgant 
(2005, p. 1) diz que: "o trabalho de Enfermagem não produz bens a serem estocados e comercializados, e sim serviços que são consumidos no ato de sua produção, isto é, no momento da assistência. essa concepção o trabalho constitui um processo".

Nesse sentido, o enfermeiro auditor, buscar-se-á averiguar todo o andamento das atividades que envolve os enfermeiros liderados, como preenchimento de prontuário, orientando os outros enfermeiros, para que as atividades ocorram de maneira profissional, ética e embasada em ação humanizada (SETZ; DINNOCENZO, 2009).

A auditoria na enfermagem deve ser compreendida como uma ação imprescindível nas atividades hospitalares, pois contribui na aquisição de informações pertinentes aos profissionais e ao funcionamento das atividades dos profissionais, além disso, há a auditoria classificada como prospectiva, ou seja, ocorre em uma ação prévia das ações dos enfermeiros, viabilizando evitar problemas administrativos, proporcionando ao paciente melhores condições de serviço da área da enfermagem, também há, a auditoria retrospectiva, sendo essa executada depois que o paciente é atendido pelos serviços hospitalares (HUSTON, 2009).

A auditoria pode ser considerada como um processo de avaliação de elevada importância para redirecionamento das ações, tendo em vista que após as análises do atendimento e identificação das deficiências podem ser feitos pareceres corretivos e/ou preventivos para restaurar essas ações (SCARPARO, 2009).

Para a sua aplicação os profissionais de enfermagem precisam compreender que sua função é indispensável, por isso, devem conhecer sua área que é a Saúde, isto é, ser um profissional competente e qualificado, conhecendo assuntos pertinentes ao seu cargo, como conceitos e práticas de enfermagem, compreender a biologia e sua constante mudança, além de buscar compreender as ações e comportamentos das pessoas, com isso, aumentando sua competência e qualificação a sua função. Portanto, no processo de auditoria em enfermagem todo o conhecimento e prática que o enfermeiro possuir o possibilitará a desenvolver sua função com influência 
positiva ao seu trabalho administrativo, dessa forma, capacitando-o, além de observar os resultados da assistência, conferindo com os objetivos planejados (SETZ e D`INNOCENZO, 2009).

O enfermeiro é o profissional habilitado para exercer a função de auditor, ou seja, diante do grupo de enfermeiros, com essa responsabilidade o enfermeiro auditor deve estar em constante qualificação, sendo assim, a abordagem desta temática é relevante, pois vem retratar questões de auditoria em enfermagem pertinentes ao desenvolvimento social e função do enfermeiro auditor, acerca desta área inovadora na enfermagem (CAMELO et al., 2009).

A função de enfermeiro tem crescido, pois a sociedade compreende sua importância. Logo, sua valorização no mercado de trabalho está em constante desenvolvimento, isto é, entende-se que o enfermeiro auditor proporciona analise as contas hospitalares e contribui para organização administrativa das unidades de saúde. Por consequência, sua função é averiguar as ações de serviço prestado, como o que foi consumido, o que deve ser recebido e administrar a atuação dos enfermeiros pertencentes ao grupo liderado pelo enfermeiro auditor.

Analisando atividades como por exemplo: preenchimento inadequado no prontuário e age na orientação à equipe de enfermagem quanto ao registro apropriado de todas as ações dos profissionais, embasando-se em princípios éticos e legais frente aos conselhos profissionais e à justiça (SETZ, 2009).

Nesse sentido, é possível qualificar a auditoria em três partes, isto é, prospectiva, que ocorre em ações denominadas como prévias, objetivando impedir possíveis problemas administrativos. O operacional será aqui classificado como ação no decorrer do serviço direcionando ao paciente ou indivíduo que necessita dos serviços da saúde. A retrospectiva possui sua atuação em ser executada após o cliente receber pelos serviços, ou seja, o paciente o cliente só terá que fazer prestação após receber todo auxílio e serviço da saúde. Logo, as três partes se completam, sendo que cada parte é necessária na função de enfermagem, o 
profissional auditor deve compreender essas partes como fundamentais ao seu cargo e executá-las com a finalidade desempenhar seu papel com destreza profissional.

\subsection{A ATUAÇAO DO INFEMEIRO AUDITOR NA ANÁLISE AS CONTAS DO AMBIENTE HOSPITALAR}

Assim, em termos de auditoria hospitalar, a complexidade desse ambiente de saúde e de seus referidos pacientes, exigem do enfermeiro prática de auditoria, assim sendo, colocar em prática saberes como técnico-científico. Nesse pensamento, usando os procedimentos cabíveis que influenciam na conta hospitalar, ou seja, na administração o enfermeiro auditor tem acesso e contribuição direta a unidade de saúde, na qual, está inserido.

Nesse mesmo sentido, a auditoria retrospectiva é vinculada a informação depois que o paciente ou cliente usufruiu dos serviços, nisso, auditoria é instruída no decorrer das ações nas diversas análises dos resultados gerados. Os resultados são alcançados através de observância dos registros e observância da atuação dos enfermeiros, com o intuito de ocasionar o avanço dos serviços prestados, ocorrendo na relação entre enfermagem e paciente (MOTTA, 2010).

À vista disso, a ação da auditoria hospitalar é estudada as peculiaridades como: ações operacionais, ações das organizações e a maneira que ocorre a ação financeira visando à qualidade da assistência prestada ao cliente. Os auditores em saúde realizam análises qualitativas e posteriormente ao faturamento, analisando possíveis anotações, elaboração de documentos, objetivando a qualidade do serviço prestado (MOTTA, 2010).

A ferramenta utilizada para averiguação pela ação de auditoria de enfermagem são os registros, ou seja, os prontuários do paciente ou cliente. Mediante, é permitido analisar qualidade do serviço prestado, classificar a necessidade da documentação além de contas hospitalares (HESS, 2010). 
A auditoria no SUS tem por finalidade auxiliar a qualidade da assistência à saúde prestada à população na premissa do cumprimento dos princípios fundamentais dos SUS: universalidade, equidade, integridade e, ainda o princípio da economicidade na utilização dos recursos financeiros envolvidos (KLUTCHOVSKY, 2009).

O Enfermeiro encarregado pela auditoria possui a responsabilidade de executar atividades como: estudos de prontuários, analisando minuciosamente seu preenchimento, preenchimento nas áreas médicas e de enfermagem, isto é, registro do cotidiano e médico e de enfermagem, tais como, história clínica, averiguação de serviços prestados, ressaltando também, que o enfermeiro auditor deve saber interagir socialmente, pois terá que liderar outros enfermeiros, ou seja, outros profissionais da área da saúde.

\section{MÉTODOS}

Em caráter de cunho bibliográfico, essa pesquisa tem por objetivo responder e mostrar "quais são as principais evidências literárias sobre a Atuação do Enfermeiro auditor frente às contas hospitalares do SUS?" Compreendendo a atuação e função do enfermeiro auditor nos setores administrativos do sistema Único de Saúde. Portanto, é importante ressaltar que a metodologia é a parte principal de uma pesquisa ou estudo de caso, na qual, é determinada uma análise minuciosa do objeto de estudo e das técnicas utilizadas nas investigações durante a pesquisa.

Para selecionar os conteúdos desse estudo, foram importantes o uso de artigos e livros, proporcionando uma compreensão do tema abordado, sendo, imprescindível para alcançar o objeto de estudo dessa pesquisa, além disso, foram utilizados quatorze artigos, publicados entre 2009 a 2018. Nesse sentido, o período de coleta dos artigos ocorreu em três meses, na qual, teve como critério de escolha artigos que retratassem a temática e viabilizassem resposta para a pergunta norteadora.

Logo, dentre os conteúdos existem: Auditoria de Enfermagem nos Hospitais e Operadoras de planos de Saúde; Tendências da função do enfermeiro auditor no 
mercado em saúde; o papel do enfermeiro na auditoria hospitalar. O levantamento desses dados foi retirado de livros, periódicos direto da base de dados em Enfermagem (BDENF).

\section{RESULTADOS E DISCUSSÃO}

Essa etapa da pesquisa mostrará os resultados e discussões que serão apresentados como os principais fatores que corroboram para a execução dessa pesquisa, a partir dos administrativos do ambiente hospitalar, mostrando eficiência e qualidade em suas fiscalizações. $O$ que se discute em relação à temática em evidência é a maneira como essa pesquisa foi conduzida.

Isto posto, para a produção desse artigo foram agrupadas algumas questões que aplicado ao trabalho determinou a base de dados desse conteúdo, trazendo os seguintes descritores: Auditoria; Enfermeiro, Contas Hospitalares. Com isso, foi criado uma tabela com alguns dos artigos que foram consultados e lidos, que contribuíram ao desenvolvimento deste trabalho, nisso a tabela I mostrará alguns artigos que foram lidos e analisados que serviram de parâmetro para o desenvolvimento, as quais estão expostas na Tabela I.

Tabela I. Tabela criada para orientação / artigos analisados, Faculdade Cidade Verde - Maringá - Paraná, (2018).

\begin{tabular}{|c|c|c|c|c|}
\hline $\begin{array}{l}\text { ESTUDO } \\
(2009 / 2018)\end{array}$ & ARTIGO & TÍTULO & OBJETIVO & DELINEAMENTO \\
\hline $\begin{array}{l}\text { Rev. Ciência } \\
\text { \& cidadania. } \\
\text { Unibave,2015 }\end{array}$ & $\begin{array}{l}\text { Maiara, } \\
\text { Wessling. } \\
\text { Ghizoni, } \\
\text { Karla } \\
\text { Pickle } \\
\text { Vunha, }\end{array}$ & $\begin{array}{l}\text { Atuação do } \\
\text { enfermeiro } \\
\text { que pratica } \\
\text { auditoria } \\
\text { hospitalar }\end{array}$ & $\begin{array}{l}\text { O objetivo } \\
\text { geral da } \\
\text { pesquisa foi } \\
\text { identificar a } \\
\text { atuação do } \\
\text { enfermeiro que }\end{array}$ & Qualitativo/Descritivo \\
\hline
\end{tabular}




\begin{tabular}{|c|c|c|c|c|}
\hline & $\begin{array}{l}\text { Kelli } \\
\text { Pazento } \\
\text { Della } \\
\text { Giustina }\end{array}$ & & $\begin{array}{l}\text { pratica a } \\
\text { auditoria } \\
\text { hospitalar em } \\
\text { um hospital de } \\
\text { grande porte. }\end{array}$ & \\
\hline $\begin{array}{l}\text { Rev. Adm. } \\
\text { Saúde vol.17, } \\
\text { № } 68 \text {, jul. - } \\
\text { Set.2017 }\end{array}$ & $\begin{array}{l}\text { Marleny, } \\
\text { Andrade } \\
\text { Abreu, } \\
\text { Vanessa } \\
\text { Estrela } \\
\text { Rolim, } \\
\text { Rosimery } \\
\text { Cruz } \\
\text { Dantas. }\end{array}$ & $\begin{array}{l}\text { O papel do } \\
\text { enfermeiro } \\
\text { frente à } \\
\text { auditoria } \\
\text { hospitalar }\end{array}$ & $\begin{array}{l}\text { O objetivo é } \\
\text { analisar a } \\
\text { produção } \\
\text { cientifica sobre } \\
\text { os registros de } \\
\text { enfermagem } \\
\text { no ambiente } \\
\text { hospitalar. }\end{array}$ & Prospectivo \\
\hline $\begin{array}{l}\text { Rev. Eletr. } \\
\text { Enf. 2013. }\end{array}$ & $\begin{array}{l}\text { Cinthya } \\
\text { Yara } \\
\text { Blanck, } \\
\text { Elizabeth } \\
\text { Navas } \\
\text { Sanches, } \\
\text { Maria } \\
\text { Tereza } \\
\text { Leopard. }\end{array}$ & $\begin{array}{l}\text { A prática do } \\
\text { enfermeiro } \\
\text { auditor } \\
\text { hospitalar } \\
\text { na região do } \\
\text { vale do } \\
\text { Itajaí }\end{array}$ & $\begin{array}{l}\text { O presente } \\
\text { estudo } \\
\text { objetivou } \\
\text { identificar a } \\
\text { atuação do } \\
\text { enfermeiro } \\
\text { auditor } \\
\text { intrahospitalar } \\
\text { na } \\
\text { mesorregião } \\
\text { do vale do } \\
\text { Itajaí/SC, e as } \\
\text { medidas à } \\
\text { destinadas } \\
\text { qualidade da } \\
\text { assistência à }\end{array}$ & $\begin{array}{l}\text { Levantamento } \\
\text { bibliográfico descritivo. }\end{array}$ \\
\hline
\end{tabular}

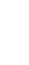




\begin{tabular}{|c|c|c|c|c|}
\hline & & & $\begin{array}{l}\text { saúde do } \\
\text { enfermo. }\end{array}$ & \\
\hline $\begin{array}{l}\text { GUGLIERI, } \\
2011\end{array}$ & $\begin{array}{l}\text { Alison } \\
\text { Carlosso } \\
\text { Guglieri. }\end{array}$ & $\begin{array}{l}\text { Os reflexos } \\
\text { econômicos } \\
\text { da auditoria } \\
\text { de } \\
\text { enfermagem }\end{array}$ & $\begin{array}{l}\text { O presente } \\
\text { estudo teve } \\
\text { como objetivo } \\
\text { identificar na } \\
\text { visão dos } \\
\text { servidores do } \\
\text { setor de } \\
\text { Gerenciamento } \\
\text { de } \\
\text { Informações e } \\
\text { AUDISUS, os } \\
\text { reflexos } \\
\text { econômicos da } \\
\text { auditoria de } \\
\text { enfermagem } \\
\text { retrospectivas } \\
\text { municipal do } \\
\text { SUS. }\end{array}$ & $\begin{array}{l}\text { Prospectivo- } \\
\text { Descritivos/Exploratório }\end{array}$ \\
\hline
\end{tabular}

Fonte: autor.

Conforme demostrado na tabela I, foi realizada uma análise documental de artigos com temas relacionados a auditoria hospitalar e as ações do enfermeiro auditor na fiscalização dos setores administrativos.

Os dois primeiros artigos analisados, que são: "Atuação do enfermeiro que pratica auditoria hospitalar" (WESLLING; GHIZONI; GIUSTINA, 2015) e "O papel do enfermeiro frente à auditoria hospitalar" (ABREU; ROLIM; DANTAS, 2017), tiveram como ideia principal conhecer a função dos enfermeiros auditores nos hospitais e suas atuações administrativas. Nisso Abreu et al. (2011) diz que: "As instituições de 
saúde com coparticipação dos profissionais auditores vêm atender as necessidades no controle orçamentário, gastos com materiais e medicamentos de alto custo, investimentos envolvidos no processo assistencial". (ABREU et al., 2017, p. 932).

Ao avaliar os artigos percebeu-se que ambos trouxeram os conteúdos pautados na atuação do enfermeiro auditor frente a auditoria hospitalar. Percebeu-se, também, que as estratégias e métodos elaborados para a atuação na fiscalização e qualificação dos enfermeiros em suas atuações foi essencial para as medidas tomadas internamente no ambiente administrativo. (WESLLING; GHIZONI; GIUSTINA, 2015; ABREU; ROLIM; DANTAS, 2017)

Nesse sentido, os dois últimos artigos da tabela I, intitulados: "A prática do enfermeiro auditor hospitalar na região do vale do Itajaí" (BLANCK; SANCHES; LEOPARD, 2017) e "Os reflexos econômicos da auditoria de enfermagem" (GUGLIERI, 2011) retratam a atuação do enfermeiro auditor e sua influência no ambiente administrativo no setor do SUS, com isso, de acordo com o autor Setz; D'innocenzo (2009, p. 314):

A auditoria na saúde é uma importante ferramenta para mensurar a qualidade do trabalho das equipes multidisciplinares, especificamente na equipe da enfermagem, na qual o enfermeiro auditor, no papel disciplinar, tem como objetivo apresentar dados das melhorias na assistência prestada, descrita nos registros de enfermagem. (SETZ; D'INNOCENZO, 2009, p. 314).

Isto é, o enfermeiro auditor, também possui outros papéis além de administrar os serviços de auditoria hospitalar, como por exemplo, liderar outros enfermeiros. Por conseguinte, os artigos que tornaram essa pesquisa uma realidade foram fundamentais para o desenvolvimento desta, pontuando as funções de enfermeiros auditores e suas abordagens no ato da auditoria hospitalar. Portanto, os profissionais que atuam nessa área, buscam em suas atividades diária pela qualidade da assistência, devendo fazer parte do cotidiano profissional. 


\section{CONCLUSÃO}

O presente artigo procurou esclarecer alguns fatores que envolvem o processo administrativo e gerencial do sistema único de saúde sendo norteado pela questão: Quais são as principais evidências literárias sobre a Atuação do Enfermeiro auditor frente às contas hospitalares do SUS? Sendo constatado que o enfermeiro auditor é o elemento chave para fiscalizar e organizar o processo operacional do ambiente hospitalar.

Entretanto, a intervenção da auditoria é fundamental, pois é através dela que se eliminam todos os meios tendenciosos que corroboram para que ocorram fraudes administrativas, além de garantir a efetividade da qualidade na prestação de serviços. Através da auditoria que se tem o controle sobre todo o processo administrativo e operacional do ambiente hospitalar do sistema único de saúde. Fazse, necessário que novas pesquisas sejam feitas em relação a temática em questão.

\section{REFERÊNCIAS}

ABREU, Marleny Andrade L. et al. Auditoria em enfermagem: revisão sistemática da literatura. Revista Brasileira de Enfermagem - REBEn, Brasília - DF, 2017.

ABREU, M. A; ROLIM, V. E.; DANTAS, R. C. O papel do enfermeiro frente à auditoria hospitalar. Rev. Adm. Saúde vol.17, № 68, jul. - Set. 2017.

BLANCK, C. Y.; SANCHES, E. N.; LEOPARD, M. T. A prática do enfermeiro auditor hospitalar na região do vale do Itajaí. Rev. Eletr. Enf., 2013.

BRASIL, Ministério da saúde. Decreto n. 4,726 de 09 de junho de 2003. Regulamenta o Departamento Nacional de Auditoria do SUS como órgão responsável pela auditoria no SUS. http://portal.saúde.gov.br/portal/arquivos/pdf/decreto4726.2003.estrutura.ms.pdf. Acesso em: 09-02-2019. 
BRASIL. Presidência da República. Lei n. 8.689 de 27 de julho de 1993. Dispõe sobe a extinção do INAMPS e dá outras providências. Disponível em: http://www2.camara.leg.br/legin/fed/lei/1993/lei-8689-27-julho-1993-362983-normapl.html. Acesso em: 19 jan. 2019.

CAMELO, S.H.H. et al. Auditoria de enfermagem e a qualidade da assistência à saúde: uma revisão de literatura. Revista eletrônica de Enfermagem, Rio de Janeiro, v.11, n. 4, out. 2009.

GUGLIERI, A. C. Os reflexos econômicos da auditoria de enfermagem. Repositório Institucional da Uninpa, 2011.

HESS, C. T. Lista de verificação de auditoria para necessidade médica de serviços prestados. Adv Skin Wound Care. 2010.

KURCGANT, P. Gerenciamento em Enfermagem. Coordenadora Paulina Kurcgant; autoras Daisy Maria Rizatto Tronchin et al. Rio de Janeiro: Guanabara Koogan, 2005.

KLUTHCOVSKY, F.A. Gestão em saúde pública. 2009. Disponível em: http://pt.scribd.com/doc/119084429/RELATO-HISTORICO-DA-AUDITORIA-E-SUAAPLICAÇÃO-NO-SUS-E-NAS-OPERADORAS-DE-SAUDE. Acesso em: 28-02-2019.

MARQUIS, Bassie L.; HUSTON, Carol J. Administração e Liderança em Enfermagem: Teoria e Prática. 6. Ed Porto Alegre: Artmed, 2009.

MOTTA, A.L.C. Auditoria de enfermagem nos hospitais e operadoras de planos de saúde. São Paulo: látria, 2010.

SCARPARO, A.F.; FERRAZ, C.A. Abordagem conceitual de métodos e finalidades da auditoria de enfermagem. Revista RENE, v. 10, n. 1, p. 124-130, jan/mar. 2009. 
SETZ, V. G.; D'INNOCENZO, M. Avaliação da qualidade dos registros de enfermagem no prontuário por meio da auditoria. Acta Paulista Enfermagem. 2009.

WESSLING, M.; GHIZONI, K. P. V.; GIUSTINA, K. P. D. Atuação do enfermeiro que pratica auditoria hospitalar. Rev. Ciência \& cidadania. Unibave, 2015.

Enviado: Setembro, 2020.

Aprovado: Setembro, 2021. 